05, 11

\title{
Сверхтвердая магнитная фаза в двумерном изингоподобном антиферромагнетике с большой одноионной анизотропией
}

\author{
(C) А.Г. Мелешко, Ф.Н. Клевец, Г.А. Гореликов, О.А. Космачев, Ю.А. Фридман ॠ \\ Крымский федеральный университет им. В.И. Вернадского, \\ Симферополь, Россия \\ ๑ E-mail: yuriifridman@gmail.com \\ (Поступила в Редакцию 14 февраля 2017 г.)
}

\begin{abstract}
Исследована модель Изинга с фрустрированным обменным взаимодействием для ультратонкой сильно анизотропной антиферромагнитной пленки в среднеполевом приближении при низкой температуре. Показано, что при определенном соотношении величин материальных констант в системе может реализоваться пространственно-неоднородное состояние наряду с однородными состояниями: ферромагнитной, квадрупольной или сверхтвердой магнитной фазой. На основе анализа линий устойчивости построена фазовая диаграмма системы.
\end{abstract}

Ф.Н. Клевец и А.Г. Мелешко благодарят фонд Дмитрия Зимина „Династия“ за финансовую поддержку. Работа выполнена при финансовой поддержке РФФИ (проекты № 16-02-00069 a, 15-42-01007 р_юг_а, 16-42910441 р_а и 16-32-00098).

DOI: 10.21883/FTT.2017.09.44842.040

\section{1. Введение}

В последнее время повышенный интерес вызывают исследования магнитных материалов, в которых имеет место конкуренция обменного взаимодействия и одноионной анизотропии. Это связанно с поиском новых квантовых состояний, существование которых было доказано теоретически для бозе-газов и которые могут реализоваться в магнетиках. В 2004 году было объявлено об экспериментальном обнаружении „сверхтвердого“ состояния ${ }^{4} \mathrm{He}[1]$. И хотя последующие исследования не подтвердили это открытие [2], работа [1] не только стимулировала разнообразные экспериментальные исследования в этом направлении, но и реанимировала работу по поиску новых квантовых состояний в магнетиках. Аналогом сверхтвердой фазы в бозе-конденсате для спиновых систем является промежуточное состояние между антиферромагнитной и спин-флоп фазой, в котором параметры порядка обеих фаз отличны от нуля, что было теоретически показано еще в 1970 г. [3]. Сорок лет спустя экспериментальные исследования газа из ионов рубидия, охлажденного до сверхнизких температур, показали, что он может находится в сверхтвердом состоянии [4]. Необходимо отметить, что термин „сверхтвердый“ описывает не твердое тело, а скорее кристалл, обладающий сверхтекучестью. В данном случае [4] газообразный рубидий распределился по ячейкам, образованным полем оптической решетки, т.е. атомы были вынуждены образовать кристалл, по сути оставаясь разреженным газом.

Помимо газов в оптических решетках сверхтвердую магнитную фазу можно обнаружить и в других квантовых магнетиках [5-10]. После того как была доказана возможность реализации сверхтвердой фазы в двухподрешеточных спиновых системах [11,12], такие системы стали одними из перспективных кандидатов для обнаружения данного состояния [11,13-19]. Например, к такого рода системам относится низкоразмерный гейзенберговский антиферромагнетик со спином единица, обладающий одноионной анизотропией [13]. Магнитными материалами, имеющими подходящую структуру и обладающими легкоплоскостной одноионной анизотропией, являются, например, $\mathrm{Ni}\left(\mathrm{C}_{2} \mathrm{H}_{8} \mathrm{~N}_{2}\right)_{2} \mathrm{NO}_{2}\left(\mathrm{ClO}_{4}\right)$ [20] и $\mathrm{Ni}\left(\mathrm{C}_{2} \mathrm{H}_{8} \mathrm{~N}_{2}\right)_{2} \mathrm{Ni}\left(\mathrm{CN}_{4}\right)$ [21]. Однако в указанных материалах сверхтвердое состояние не реализуется, поскольку одним из условий реализации сверхтвердой магнитной фазы является наличие в системе большой легкоплоскостной одноионной анизотропии, в то время как указанные материалы являются слабо анизотропными, т. е. при температурах, меньших температуры Нееля, они находятся в спонтанно упорядоченном состоянии. Во внешнем магнитном поле магнитные моменты в таких системах ориентируются по полю, и уже при достаточно малых полях в них реализуется ферромагнитное упорядочение.

Материалами, обладающими большой одноионной анизотропией, являются, например, антиферромагнетики $\mathrm{CsFeBr}_{3}$ [22,23], $\mathrm{RbFeBr}_{3}$ [24] и $\mathrm{CsFeCl}_{3}$ [25]. В таких магнетиках квантовые свойства отдельных спинов в эффективном магнитном поле играют решающую роль в формировании динамических и термодинамических свойств $[26,27]$. Влияние большой одноионной анизотропии проявляется, в том числе, в квантовом сокращении спина, приводящем к тому, что среднее значение намагниченности (векторный параметр порядка) на узле равно нулю, и в системе реализуется дальний магнитный порядок тензорного типа - квадрупольная фаза. В этой фазе параметры порядка системы образуют тензор квадрупольных моментов, в отличие от векторных параметров порядка, характеризующих магнитное упорядочение 
в дипольных фазах (ферромагнитной, антиферромагнитной и т.п.).

Также одним из перспективных кандидатов на обнаружение сверхтвердой магнитной фазы являются фрустрированные магнетики $[28,29]$. Простейшим примером такой системы может быть двухподрешеточный антиферромагнетик, в котором обменное взаимодействие между магнитными ионами в подрешетке отличается от межподрешеточного обменного взаимодействия. Как было показано ранее [13,28-32], в такой системе могут реализовываться разнообразные фазовые состояния: спиновая жидкость, сверхтвердая фаза, магнитные плато. Если рассматривать частный случай двухподрешеточного изинговского антиферромагнетика, которому соответствует, например $\mathrm{Ba}_{2} \mathrm{CoGe}_{2} \mathrm{O}_{7}$, то все перечисленные фазовые состояния могут быть обнаружены в нем $[29,30]$, однако области их существования и типы фазовых переходов могут существенным образом отличаться. Кроме того, очевидно, что реализация указанных выше состояний существенно зависит от величины и типа одноионной анизотропии [13,29-32].

Существует довольно много исследований сверхтвердой магнитной фазы для трехмерных двухподрешеточных антиферромагнетиков с большой легкоплоскостной одноионной анизотропией $[12,13,29-35]$, т.е. без учета магнитодипольного взаимодействия. Однако магнитодипольное взаимодействие оказывает существенное влияние не только в обычных кристаллах, но и в спиновых конденсатах [36]. Такие системы (с существенным магнитодипольным взаимодействием) являются перспективными с точки зрения квантовой обработки информации [37,38] и квантовых вычислений [39]. К сожалению, нам не удалось найти работ, посвященных исследованию сверхтвердой магнитной фазы в двумерных системах. Очевидно, двумерность системы подразумевает существенное влияние магнитодипольного взаимодействия, которое может усиливать легкоплоскостную анизотропию и приводить к реализации пространственно-неоднородных состояний, например плоскопараллельных доменов [40-42] и вихревых структур [43-45]. При этом данные состояния могут реализовываться не только в ферромагнетиках, но также и в антиферромагнетиках [44-47].

Целью данной работы является исследование фазовых состояний и спектров элементарных возбуждений в ультратонкой (двумерной) сильно анизотропной антиферромагнитной пленке со спином единица и фрустрированным изингоподобным обменным взаимодействием (рис. 1) в области низких температур в приближении среднего поля. Мы рассматриваем случай, когда обменное взаимодействие приводит к ферромагнитному упорядочению в подрешетках, в то время как межподрешеточное обменное взаимодействие - антиферромагнитного типа. Вопрос о возможности использования приближения среднего поля в данном случае требует дополнительного обсуждения, которое будет приведено ниже в тексте статьи.

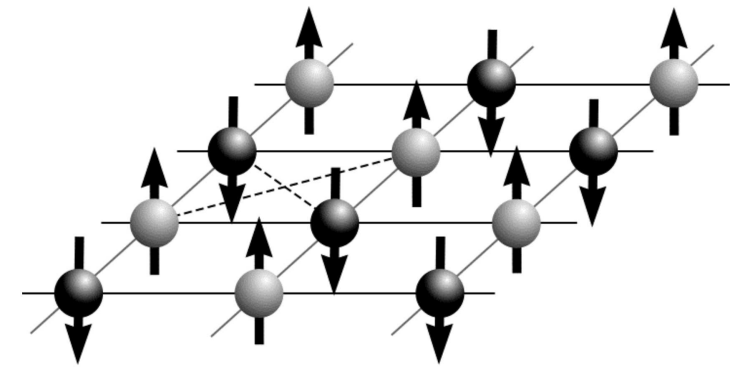

Рис. 1. Простая квадратная решетка двумерного двухподрешеточного изингоподобного антиферромагнетика со слабой одноионной анизотропией в отсутствие внешнего магнитного поля. Сплошные линии показывают расстояние между узлами в разных подрешетках, штриховые линии - расстояние между узлами в одной подрешетке.

\section{2. Модель}

Кристаллическая решетка рассматриваемой системы показана схематически на рис. 1 . Как видно из рис. 1 , наша модель описывает двумерную систему с эквивалентными подрешетками, состоящими из магнитных ионов со спином единица, формирующих шахматное упорядочение. Такая система может рассматриваться как двумерный двухподрешеточный антиферромагнетик. Расстояние между соседними подрешетками меньше, чем между соседними узлами в подрешетке, в результате чего энергия обменного взаимодействия между подрешетками больше, чем энергия обменного взаимодействия внутри подрешетки, что приводит к антиферромагнитному упорядочению.

Единичный спин является тем минимальным значением спина, для которого возможно существование одноосной одноионной анизотропии. В данном исследовании мы выбрали для рассмотрения анизотропию типа „легкая плоскость“, с базисной плоскостью ХOY. Внешнее магнитное поле перпендикулярно базисной плоскости, т. е. параллельно оси $O Z$. Данную модель можно описать следующим гамильтонианом:

$$
\begin{aligned}
\mathscr{H}= & -\frac{1}{2} \sum_{n, n^{\prime}} J_{n n^{\prime}} S_{n}^{z} S_{n^{\prime}}^{z}-\frac{1}{2} \sum_{n, n^{\prime}} V_{n n^{\prime}}^{i j} S_{n}^{i} S_{n^{\prime}}^{j}+\frac{1}{2} \sum_{n, m} \tilde{J}_{n m} S_{n}^{z} S_{m}^{z} \\
& -\frac{1}{2} \sum_{n, m} \tilde{V}_{n m}^{i j} S_{n}^{i} S_{m}^{j}+D \sum_{n}\left(S_{n}^{z}\right)^{2}-H \sum_{n} S_{n}^{z},
\end{aligned}
$$

где $J_{n n^{\prime}}$ и $\tilde{J}_{n m}-$ подрешеточный (ферромагнитный) и межподрешеточный (антиферромагнитный) обменные интегралы, соответственно, причем $J_{n n^{\prime}}<\tilde{J}_{n m}, V_{n n^{\prime}}^{i j}$ и $\tilde{V}_{n m}^{i j}$ - компоненты тензоров подрешеточного и межподрешеточного магнитодипольного взаимодействия, $D-$ константа одноионной анизотропии, $H-$ внешнее магнитное поле в энергетических единицах, $S_{n}^{i}-i$-я проекция оператора спина в узле $n$. Фурье-компоненты тензоров магнитодипольного взаимодействия внутри подре- 
шетки имеют вид [48]

$$
\begin{gathered}
V_{k}^{x x}=\frac{A_{0}}{3}-\Omega_{0} k \cos ^{2} \psi, \quad V_{k}^{y y}=\frac{A_{0}}{3}-\Omega_{0} k \sin ^{2} \psi, \\
V_{k}^{z z}=-\frac{2}{3} A_{0}+\Omega_{0} k, \quad V_{k}^{x y}=V_{k}^{y x}=-\frac{\Omega_{0}}{2} k \sin 2 \psi, \\
V_{k}^{x z}=V_{k}^{z x}=V_{k}^{y z}=V_{k}^{z y}=0,
\end{gathered}
$$

где $A_{0}=\frac{3}{2}\left(g \mu_{B}\right)^{2} \sum_{R \neq 0} R^{-3}$ и $\Omega_{0}=\frac{2 \pi}{a^{2}}\left(g \mu_{B}\right)^{2}-$ параметры магнитодипольного взаимодействия, $g$ - гиромагнитное отношение, $\mu_{B}-$ магнетон Бора, $a^{2}-$ „объем“ плоской элементарной ячейки в подрешетке, $k$ - волновой вектор, $\psi-$ угол, образуемый волновым вектором с осью $O X$ в базисной плоскости $X O Y$. Такой выбор способа задания ориентации волнового вектора в базисной плоскости произволен и не снижает общности рассматриваемой задачи. Он был сделан с единственной целью упростить последующие вычисления. Выражения для Фурье-компонент тензора магнитодипольного взаимодействия между подрешетками имеют вид, аналогичный (2), с учетом замены параметров магнитодипольного взаимодействия на $\tilde{A}_{0}$ и $\Omega_{0}$, характеризующие межподрешеточное магнитодипольное взаимодействие. Причем, как было отмечено выше, имеют место следующие соотношения: $\tilde{A}_{0}>A_{0}$ и $\tilde{\Omega}_{0}>\Omega_{0}$.

В дальнейшем мы предполагаем, что энергия обменных взаимодействий много меньше энергии одноионной анизотропии. Таким образом, имеет место следующее соотношение материальных параметров системы: $D \gg \tilde{J}_{0}>J_{0} \gg \tilde{A}_{0}>A_{0}$. При этом величина внешнего магнитного поля является варьируемым параметром. Кроме того, мы рассматриваем случай низких температур $(T \rightarrow 0)$.

\section{3. Однородные состояния}

Предположим, что внешнее магнитное поле настолько велико, что зеемановская энергия преобладает над энергиями других взаимодействий $\left(H \gg D \gg \tilde{J}_{0}>J_{0} \gg\right.$ $\left.\gg \tilde{A}_{0}>A_{0}\right)$. Очевидно, что в таком случае магнитные моменты всех узлов ориентируются вдоль поля, т.е. поведение системы можно свести к поведению ферромагнетика. Выделяя среднее поле, гамильтониан (1) можно представить в следующем виде: $\mathscr{H}=\mathscr{H}_{0}+\mathscr{H}_{\text {int }}$, где $\mathscr{H}_{\text {int }}$ - гамильтониан взаимодействия, а $\mathscr{H}_{0}-$ одноузельный гамильтониан, который в данном случае имеет следующий вид:

$$
\begin{aligned}
\mathscr{H}_{0}=-\left\{H+\left[J_{0}-\frac{2}{3} A_{0}\right.\right. & \left.\left.-\frac{1}{2}\left(\tilde{J}_{0}+\frac{2}{3} \tilde{A}_{0}\right)\right]\left\langle S^{z}\right\rangle\right\} \\
& \times \sum_{n} S_{n}^{z}+D \sum_{n}\left(S_{n}^{z}\right)^{2} .
\end{aligned}
$$

В рассматриваемом случае использование приближения среднего поля вполне обосновано, поскольку мы рассматриваем случай низких температур, когда температурные флуктуации малы и практически не влияют на состояние системы. Что же касается квантовых флуктуаций, то они конечны, поскольку сходимость интеграла флуктуаций для всех однородных состояний обеспечива- ется учетом магнитодипольного взаимодействия [48-51] и влиянием внешнего магнитного поля. Поэтому теорема Мермина-Вагнера о реализации дальнего магнитного порядка в двумерных системах не применима для рассматриваемой модели. Таким образом, можно утверждать, что приближение среднего поля можно использовать при рассмотрении данной модели. Кроме того, как было доказано в [26,27], приближение среднего поля можно использовать при рассмотрении систем любой размерности. Также необходимо отметить работу [5], где было показано, что приближение среднего поля дает правильные результаты для модели Гейзенберга с фрустрированным обменным взаимодействием для спина $1 / 2$, в частности при исследовании сверхтвердой магнитной фазы.

Решая уравнение Шредингера с гамильтонианом (3), найдем уровни энергии магнитного иона и его собственные функции

$$
\begin{gathered}
E_{1,-1}=\mp H+D \mp\left[J_{0}-\frac{2}{3} A_{0}-\frac{1}{2}\left(\tilde{J}_{0}+\frac{2}{3} \tilde{A}_{0}\right)\right]\left\langle S^{z}\right\rangle ; \\
E_{0}=0 ; \\
|\Psi(1)\rangle=|1\rangle ; \quad|\Psi(0)\rangle=|0\rangle ; \quad|\Psi(-1)\rangle=|-1\rangle .
\end{gathered}
$$

Как видно из (4), когда энергия внешнего магнитного поля превосходит энергии всех других взаимодействий в системе, нижайшим энергетическим уровнем является $E_{1}$, а, следовательно, $|1\rangle-$ основное состояние системы. На базисе собственных функций системы построим операторы Хаббарда $X^{M M^{\prime}}=|\Psi(M)\rangle\left\langle\Psi\left(M^{\prime}\right)\right|$, описывающие переход магнитного иона из состояния $M$ в состояние $M^{\prime}$ [51-54]. В рассматриваемом случае сильного внешнего магнитного поля спиновые операторы связаны с операторами Хаббарда простыми соотношениями

$$
\begin{gathered}
S^{z}=X^{11}-X^{-1-1} ; \\
S^{+}=\sqrt{2}\left(X^{10}+X^{0-1}\right) ; \quad S^{-}=\left(S^{+}\right)^{\dagger} .
\end{gathered}
$$

При этом среднее значение намагниченности на узле $\left\langle S^{z}\right\rangle=1$. Такое значение параметра порядка характерно для ферромагнитного упорядочения, реализация которого обусловлена сильным внешним полем.

Чтобы определить границы существования ферромагнитного состояния, исследуем его динамические особенности, а именно рассмотрим спектры элементарных возбуждений, которые можно найти, рассмотрев полюса функции Грина системы [52-54]: $G^{\alpha \beta}\left(n, \tau, n^{\prime}, \tau^{\prime}\right)=$ $=-\left\langle\hat{T} \tilde{X}_{n}^{\alpha}(\tau) \tilde{X}_{n^{\prime}}^{\beta}\left(\tau^{\prime}\right)\right\rangle$ где $\alpha \quad$ и $\beta-$ так называемые корневые векторы, определяемые алгеброй операторов Хаббарда $[12,53,55], \hat{T}-$ оператор Вика, $\tilde{X}_{n}^{\alpha}(\tau)=e^{\mathscr{H} \tau} X_{n}^{\alpha} e^{-\mathscr{H} \tau}-$ оператор Хаббарда в представлении взаимодействия, $\mathscr{H}=\mathscr{H}_{0}+\mathscr{H}_{\text {int }}-$ полный гамильтониан системы. В терминах операторов Хаббарда одноузельный гамильтониан имеет диагональный вид $\mathscr{H}_{0}=\sum_{M} E_{M} X_{n}^{M M}$, а гамильтониан взаимодействия можно представить следующим образом:

$$
\mathscr{H}_{\text {int }}=-\frac{1}{2} \sum_{\substack{n, n^{\prime} \\ \alpha, \beta}}\left\{\mathbf{c}(\alpha) \hat{B}_{n n^{\prime}} \mathbf{c}(\beta)\right\} X_{n}^{\alpha} X_{n^{\prime}}^{\beta},
$$


где с - вектор, компоненты которого определяются из связи спиновых операторов с операторами Хаббарда (6), а матрица $\hat{B}_{n n^{\prime}}-$ блочная матрица, $\hat{B}_{n n^{\prime}}=\hat{B}_{11} \otimes \hat{B}_{22}$, где $\hat{B}_{11}=J_{n n^{\prime}}-\frac{\tilde{J}_{n n^{\prime}}}{2}+V_{n n^{\prime}}^{z z}+\tilde{V}_{n n^{\prime}}^{z z}$, a

$$
\hat{B}_{22}=\left(\begin{array}{cc}
V_{n n^{\prime}}^{x x}+\tilde{V}_{n n^{\prime}}^{x x}-V_{n n^{\prime}}^{y y}-\tilde{V}_{n n^{\prime}}^{y y}-2 i\left(V_{n n^{\prime}}^{x y}+\tilde{V}_{n n^{\prime}}^{x y}\right) & V_{n n^{\prime}}^{x x}+\tilde{V}_{n n^{\prime}}^{x x}+V_{n n^{\prime}}^{y y}+\tilde{V}_{n n^{\prime}}^{y y} \\
V_{n n^{\prime}}^{x x}+\tilde{V}_{n n^{\prime}}^{x x}+V_{n n^{\prime}}^{y y}+\tilde{V}_{n n^{\prime}}^{y y} & V_{n n^{\prime}}^{x x}+\tilde{V}_{n n^{\prime}}^{x x}-V_{n n^{\prime}}^{y y}-\tilde{V}_{n n^{\prime}}^{y y}+2 i\left(V_{n n^{\prime}}^{x y}+\tilde{V}_{n n^{\prime}}^{x y}\right)
\end{array}\right) .
$$

Решение дисперсионного уравнения для ферромагнитной фазы позволяет найти спектр элементарных возбуждений, который в длинноволновом пределе имеет следующий вид:

$$
\varepsilon_{F M}(k)=\sqrt{\begin{array}{c}
\left(H-D+J_{0}-\frac{\tilde{J}_{0}}{2}-A_{0}-\frac{2}{3} \tilde{A}_{0}\right)^{2}+ \\
+\left(H-D+J_{0}-\frac{\tilde{J}_{0}}{2}-A_{0}-\frac{2}{3} \tilde{A}_{0}\right)\left(\Omega_{0}+\tilde{\Omega}_{0}\right) k
\end{array}} .
$$

Прежде всего отметим, что в спектре (8) имеется корневая зависимость от волнового вектора, обусловленная учетом магнитодипольного взаимодействия. Такая зависимость приводит к сходимости интеграла флуктуаций, a, следовательно, и к стабилизации дальнего магнитного порядка в двумерной системе. Очевидно, спектр (8) становится бездисперсионным, если пренебречь магнитодипольным взаимодействием, что характерно для систем, в которых учитывается только изингоподобное обменное взаимодействие. Необходимо отметить, что если внешнее магнитное поле нарушает симметрию системы, например поле перпендикулярно оси $O Z$ (что не соответствует рассматриваемому случаю, так как поле параллельно оси $O Z$ ), то изинговское обменное взаимодействие также может приводить к дисперсии магнонов [56].

Кроме того, видно, что энергия магнонов не зависит от ориентации волнового вектора и принимает минимальное значение при $k=0$. Линия устойчивости ферромагнитной фазы определяется из обращения в ноль энергетической щели в спектре (8) и имеет вид

$$
H_{F M}^{C}=D-J_{0}+\frac{\tilde{J}_{0}}{2}+A_{0}+\frac{2}{3} \tilde{A}_{0} .
$$

Таким образом, учет магнитодипольного взаимодействия изменяет закон дисперсии элементарных возбуждений в ферромагнитной фазе, однако не приводит к возникновению неоднородного состояния. Т.е. влияние магнитодипольного взаимодействия сводится к статической перенормировке линии устойчивости ферромагнитной фазы - уменьшению области существования ферромагнитного состояния по сравнению с результатами, полученными для трехмерных моделей без учета магнитодипольного взаимодействия [33].

Теперь исследуем случай, когда энергия внешнего магнитного поля много меньше энергии одноионной анизотропии $(H \ll D)$. В этом случае магнитным моментам энергетически выгодно ориентироваться в базисной плоскости. Одноузельный гамильтониан в этом состоянии совпадет с одноузельным гамильтонианом (3). Реше- ние уравнения Шредингера дает энергетический спектр магнитного иона (4) и собственные функции (5). Однако в исследуемом случае нижайшим энергетическим уровнем является $E_{0}$, т.е. в системе происходит инверсия энергетических уровней. При этом состояние $|0\rangle$ является основным состоянием системы. Это приводит к тому, что среднее значение намагниченности на один узел равно нулю, $\left\langle S^{z}\right\rangle=0$. Таким образом мы получили новое состояние с нулевой намагниченностью, не являющееся при этом парамагнитным, поскольку для него отличны от нуля компоненты тензора квадрупольных моментов, и параметры порядка имеют следующий вид:

$$
\left\langle S^{z}\right\rangle=0 ; \quad q_{2}^{0}=-2 ; \quad q_{2}^{2}=q_{2}^{x y}=q_{2}^{x z}=q_{2}^{y z}=0,
$$

где $q_{2}^{i}=\left\langle O_{2 n}^{i}\right\rangle, O_{2 n}^{0}=3\left(S_{n}^{z}\right)^{2}-S(S+1), O_{2 n}^{2}=\left(S_{n}^{x}\right)^{2}-\left(S_{n}^{y}\right)^{2}$, $O_{2 n}^{i j}=\left[S_{n}^{i}, S_{n}^{j}\right]_{+}(i, j=x, y, z ; i \neq j)$ - операторы Стивенса [57]. Это так называемое квадрупольное состояние $[58,59]$, описываемое тензорными параметрами порядка. Геометрическим образом этого состояния является эллипс, главная ось которого лежит в плоскости $X O Y$.

Подставляя (10) в (4), можно получить энергетические уровни магнитного иона в квадрупольной фазе в более простом виде

$$
E_{ \pm 1}=D \mp H ; \quad E_{0}=0 .
$$

Решая дисперсионное уравнение в квадрупольном состоянии, найдем спектр элементарных возбуждений, который в длинноволновом пределе имеет следующий вид:

$$
\begin{gathered}
\varepsilon_{Q U}^{2}(k)=D^{2}+H^{2}-4 D\left[\frac{2}{3}\left(A_{0}+\tilde{A}_{0}\right)+\left(\Omega_{0}+\tilde{\Omega}_{0}\right) k\right] \\
-2 \sqrt[\begin{array}{r}
H^{2} D\left[D-\frac{8}{3}\left(A_{0}+\tilde{A}_{0}\right)+4\left(\Omega_{0}+\tilde{\Omega}_{0}\right) k\right]+ \\
+\frac{4}{9} D^{2}\left(A_{0}-\tilde{A}_{0}\right)\left[\left(A_{0}-\tilde{A}_{0}\right)+3\left(\Omega_{0}-\tilde{\Omega}_{0}\right) k\right]
\end{array}]{ }
\end{gathered}
$$

Видно, что спектр (12) имеет корневую зависимость от волнового вектора и не зависит от его ориентации. Причем, как и в ферромагнитной фазе, зависимость от волнового вектора возникает благодаря учету магнитодипольного взаимодействия. Минимум энергии элементарных возбуждений наблюдается при нулевом значении $k$. Линию устойчивости квадрупольного состояния получим из обращения в ноль энергетической щели в спектре (12)

$$
H_{Q U}^{C}=D-\frac{2}{3}\left(A_{0}+3 \tilde{A}_{0}\right)
$$

В данном случае, как и в случае сильного магнитного поля, влияние магнитодипольного взаимодействия также 
не приводит к появлению пространственно-неоднородных фазовых состояний, но проявляется в статической перенормировке спектров, уменьшая область существования квадрупольной фазы, по сравнению с результатами, полученными для объемных систем [33].

\section{4. Сверхтвердая магнитная фаза}

Теперь рассмотрим случай промежуточных полей, когда $H_{Q U}^{C}<H<H_{F M}^{C}$. В этом случае конкуренция легкоплоскостной анизотропии, усиливаемой магнитодипольным взаимодействием, внешнего магнитного поля и обменных взаимодействий приводит к тому, что магнитные моменты подрешеток ориентированы под некоторым углом к направлению внешнего поля. Предположим, что в разных подрешетках отклонение магнитных моментов от оси квантования различно, т. е. система находится не в спин-флоп фазе, а в сверхтвердом состоянии $[4,12,31,32]$.

Введем системы координат, привязанные к подрешеткам (рис. 2). Тогда намагниченность первой подрешетки будет составлять некоторый угол $\vartheta_{1}$ с направлением внешнего магнитного поля (т. е. осью $O Z$ ), а намагниченность второй подрешетки - угол $\vartheta_{2}$ с осью OZ. Для простоты будем считать, что намагниченности подрешеток лежат в плоскости XOZ. Это упрощает рассмотрение, но совершенно не влияет на общность рассматриваемой задачи.

Исследование данного состояния удобно проводить в подвернутой системе координат, когда намагниченности подрешеток ориентированы вдоль оси $O Z$. Повернем систему координат, связанную с первой подрешеткой, на угол $\vartheta_{1}$ вокруг оси $O Y$, а систему координат, связанную со второй подрешеткой - на угол $\vartheta_{2}$. При этом гамильтониан (1) можно представить в виде суммы двух слагаемых, одно из которых диагонально в терминах операторов Хаббарда (6), $\mathscr{H}_{\text {diag }} \sim X^{M M}$, а второе содержит члены, пропорциональные недиагональным операторам Хаббарда, $\mathscr{H}_{\text {nondiag }} \sim X^{M M^{\prime}}$.

В данном случае удобно диагонализовать гамильтониан, используя метод бозонизации операторов Хаббарда [60]. Основная идея этого метода состоит в том, чтобы построить бозевский аналог гамильтониана системы, т.е. некоторым образом связать операторы Хаббарда с операторами рождения и уничтожения так, чтобы определенная часть матричных элементов совпадала с матричными элементами гамильтониана (1). Однако проблема состоит в том, что размерность физического пространства операторов Хаббарда равна трем (для $S=1$ ), тогда как гильбертово пространство операторов рождения и уничтожения бесконечномерное. Эту проблему удается решить путем построения псевдохаббардовских операторов, которые действуют в бесконечномерном гильбертовом пространстве состояний и определенным образом связаны как с операторами рождения и уничтожения квазичастиц, так и с операторами Хаббарда [60]. Используя данный подход, мы можем представить гамильтониан (1) через бозе-операторы

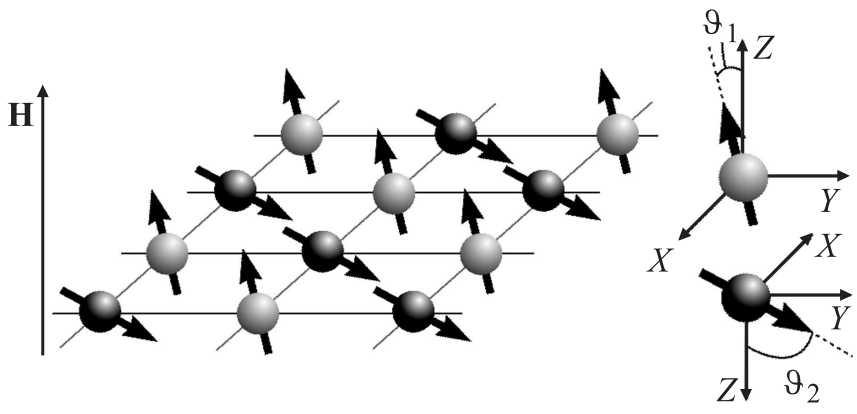

Рис. 2. Ориентация магнитных моментов подрешеток в сверхтвердой магнитной фазе.

рождения и уничтожения, в результате чего для $i$-той подрешетки получим

$$
\mathscr{H}_{i}=\mathscr{H}_{i}^{(1)}+\mathscr{H}_{i}^{(2)}
$$

где $\mathscr{H}_{i}^{(1)}$ содержит только слагаемые, линейные по операторам рождения и уничтожения магнонов, а $\mathscr{H}_{i}^{(2)}$ представляет собой гамильтониан идеального газа магнонов. Поскольку среднее от линейных слагаемых обращается в ноль, то они не несут никакого физического смысла, и можно положить амплитуды при этих слагаемых равными нулю, что приводит к системе уравнений на равновесное значение углов ориентации магнитных моментов подрешеток $\vartheta_{1}$ и $\vartheta_{2}$

$$
\left\{\begin{array}{l}
H-D \cos \vartheta_{1}+\left(J_{0}-A_{0}\right) \cos ^{2} \vartheta_{1} \\
+\frac{1}{2}\left(\tilde{J}_{0}-\frac{2}{3} \tilde{A}_{0}-\frac{\tilde{A}_{0} \sin \vartheta_{2}}{6 \sin \vartheta_{1}}\right) \cos \vartheta_{1} \cos \vartheta_{2}=0 \\
H-D \cos \vartheta_{2}+\left(J_{0}-A_{0}\right) \cos ^{2} \vartheta_{2} \\
-\frac{1}{2}\left(\tilde{J}_{0}+\frac{2}{3} \tilde{A}_{0}+\frac{\tilde{A}_{0} \sin \vartheta_{1}}{6 \sin \vartheta_{2}}\right) \cos \vartheta_{1} \cos \vartheta_{2}=0
\end{array}\right.
$$

Диагонализуя гамильтониан $\mathscr{H}_{i}^{(2)}$ с помощью стандартного $u-v$ преобразования [61], получим

$$
\mathscr{H}_{i}^{(2)}=\sum_{k} \varepsilon_{S S_{1}}^{i}(k) \alpha_{k}^{\dagger} \alpha_{k}+\sum_{k} \varepsilon_{S S_{2}}^{i}(k) \beta_{k}^{\dagger} \beta_{k},
$$

где $\varepsilon_{S S_{1}}^{i}$ и $\varepsilon_{S S_{2}}^{i}-$ спектры низкочастотных и высокочастотных магнонов для $i$-ой подрешетки, $\alpha$ и $\alpha^{\dagger}-$ бозе-операторы, соответствующие переходу иона из состояния $E_{1}$ в состояние $E_{0}$, и наоборот, а $\beta$ и $\beta^{\dagger}-$ соответствуют переходу из состояния $E_{1}$ в состояние $E_{-1}$, и наоборот. Точный вид этих спектров невозможно найти аналитически, но можно найти выражения для спектров по теории возмущений вблизи линий устойчивости ферромагнитного состояния. Вблизи этой линии 
низкочастотный спектр для $i$-ой подрешетки имеет вид

$$
\begin{aligned}
& \left(\varepsilon_{S S_{1}}^{i}\right)^{2}=\left\{H-D+J_{0}+\frac{(-1)^{i+1}}{2} \tilde{J}_{0}-A_{0}-\frac{2}{3} \tilde{A}_{0}\right. \\
& +\frac{1}{4}\left[\left(\Omega_{0} \cos \vartheta_{i}-\tilde{\Omega}_{0} \cos \vartheta_{i+1}\right) \cos \vartheta_{i} \cos ^{2} \psi\right. \\
& \left.\left.+3\left(\Omega_{0}+\tilde{\Omega}_{0}\right) \sin ^{2} \psi\right] k+\frac{1}{8}\left[J_{0} \sin \vartheta_{i}+(-1)^{i} \tilde{J}_{0} \sin \vartheta_{i}\right] k^{2}\right\} \\
& \times\left\{H-D+J_{0}+\frac{(-1)^{i+1}}{2} \tilde{J}_{0}-A_{0}-\frac{\tilde{A}_{0}}{6}\right. \\
& +\frac{1}{4}\left[3\left(\Omega_{0} \cos \vartheta_{i}-\tilde{\Omega}_{0} \cos \vartheta_{i+1}\right) \cos \vartheta_{i} \cos ^{2} \psi\right. \\
& \left.\left.+\left(\Omega_{0}+\tilde{\Omega}_{0}\right) \sin ^{2} \psi\right] k+\frac{3}{8}\left(J_{0} \sin \vartheta_{i}+(-1)^{i} \tilde{J}_{0} \sin \vartheta_{i+1}\right) k^{2}\right\}
\end{aligned}
$$

Рассмотрим эти спектры вблизи линии фазового перехода в ферромагнитную фазу, когда $\vartheta_{i}$ практически равны нулю. В этом случае линия потери устойчивости сверхтвердой фазы определяется уравнением (17) для второй подрешетки $(i=2)$

$$
H_{S S}^{C}=D-J_{0}+\frac{\tilde{J}_{0}}{2}+A_{0}+\frac{2}{3} \tilde{A}_{0}
$$

Видно, что выражения (9) и (18) совпадают, что указывает на то, что фазовый переход из сверхтвердого состояния в ферромагнитную фазу является фазовым переходом второго рода, а поле (9) или (18) - это поле фазового перехода. Это также видно из системы уравнений (15). Угол $\vartheta_{2}$ отклонения намагниченности от внешнего магнитного поля становится равным нулю при $H=H_{F M}^{C}$, в то время как намагниченность первой подрешетки ориентируется по полю раньше.

Очевидно, что спектры (17) существенно зависят от угла ориентации волнового вектора в базисной плоскости $\psi$. Так, в зависимости от величины угла $\psi$, знак линейного по волновому вектору слагаемого может меняться. Причем, когда это слагаемое будет отрицательным, мы получим так называемый „неоднородный“ спектр элементарных возбуждений [40] - знак при линейных и квадратичных по волновому вектору слагаемых будет разным, в результате чего минимуму энергии элементарных возбуждений соответствует не $k=0$, а некоторое критическое значение $k=k^{*}$, которое для спектра (17) имеет вид

$$
\begin{aligned}
k_{i}^{*}= & {\left[H-D+J_{0}+\frac{(-1)^{i+1}}{2} \tilde{J}_{0}-A_{0}-\frac{7}{12} \tilde{A}_{0}\right] } \\
& \times\left(\tilde{\Omega}_{0} \cos 2 \psi-\Omega_{0}\right) /\left\{\left[\tilde{J}_{0}+(-1)^{i} J_{0}\right] \sin ^{2} \vartheta_{i}\right. \\
& \left.+\frac{3\left(\Omega_{0}-\tilde{\Omega}_{0} \cos 2 \psi\right)^{2}+\left(\Omega_{0}^{2}-\tilde{\Omega}_{0}^{2}\right) \sin ^{2} 2 \psi}{4\left(H-D+J_{0}+\frac{\tilde{J}_{0}}{2}-A_{0}-\frac{7}{12} \tilde{A}_{0}\right)}\right\} .
\end{aligned}
$$

Из соотношения (19) следует, что период неоднородности, обратно пропорциональный $k^{*}$, существенно зависит не только от угла ориентации волнового вектора, но и от соотношений между параметрами магнитодипольного взаимодействия. Анализ этого выражения показывает, что критическое значение волнового вектора $k_{i}^{*}$ для обеих подрешеток принимает положительное значение, если $\Omega_{0}<\tilde{\Omega}_{0} \cos 2 \psi$. Из этого соотношения легко получить условие на угол ориентации волнового вектора в базисной плоскости, при выполнении которого $k_{i}^{*}>0$

$$
\cos 2 \psi>\frac{\Omega_{0}}{\tilde{\Omega}_{0}}, \quad \tilde{\Omega}_{0} \geq \Omega_{0} .
$$

Таким образом, при выполнении условия (20) минимум энергии элементарных возбуждений (17) наблюдается при отличных от нуля значениях волнового вектора, при этом система находится в неоднородной магнитной фазе с периодом неоднородности $1 / k^{*}$. Чтобы найти линию устойчивости сверхтвердого магнитного состояния вблизи ферромагнитной фазы, положим энергетическую щель в спектрах (17) равной нулю. Учитывая (19), получаем для первой и второй подрешеток соответственно

$$
\begin{aligned}
H_{I N_{1}}^{C}= & D-J_{0}-\frac{\tilde{J}_{0}}{2}+A_{0}+\frac{2}{3} \tilde{A}_{0} \\
& -\frac{1}{2 \sqrt{2}} \sqrt{\frac{D-J_{0}+A_{0}}{\tilde{J}_{0}-J_{0}}}\left(\tilde{\Omega}_{0} \cos 2 \psi-\Omega_{0}\right), \\
H_{I N_{2}}^{C}= & D-J_{0}+\frac{\tilde{J}_{0}}{2}+A_{0}+\frac{2}{3} \tilde{A}_{0} \\
& -\frac{1}{2 \sqrt{2}} \sqrt{\frac{D-J_{0}+A_{0}}{\tilde{J}_{0}+J_{0}}}\left(\tilde{\Omega}_{0} \cos 2 \psi-\Omega_{0}\right) .
\end{aligned}
$$

Видно, что $H_{I N_{1}}^{C}<H_{I N_{2}}^{C}$, т.е. вторая подрешетка переходит в пространственно-неоднородное состояние при больших полях. Следовательно, поле перехода из ферромагнитной фазы в неоднородное состояние определяется выражением (22) с периодом неоднородности $1 / k_{2}^{*}$.

Реализация пространственно-неоднородной фазы в рассматриваемой модели определятся $z$-ой проекцией намагниченности: чем больше эта проекция намагниченности, тем больше влияние магнитодипольного взаимодействия, определяющего реализацию пространственно-неоднородной магнитной фазы. Однако увеличение внешнего магнитного поля (и, следовательно, z-ой проекции намагниченности) приводит к фазовому переходу из пространственно-неоднородной фазы в однородную сверхтвердую магнитную фазу при $H>H_{I N_{2}}^{C}$ из-за конкуренции между внешним магнитным полем и легкоплоскостной анизотропией совместно с изингоподобным обменным взаимодействием. В этом случае линия устойчивости (22) реализуется вблизи ферромагнитной фазы - как видно из выражений (9) и (22), значения $H_{F M}^{C}$ и $H_{I N_{2}}^{C}$ близки. При этом вопрос о фазовых состояниях вблизи линии устойчивости квадрупольной фазы (13) остается открытым. Магнитный мо- 


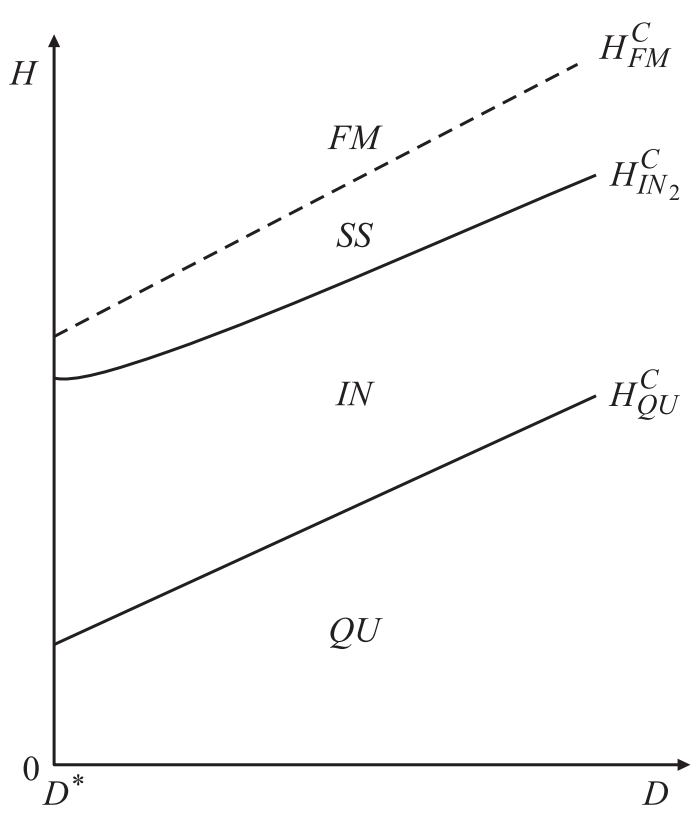

Рис. 3. Качественная фазовая диаграмма исследуемой системы. FM обозначает ферромагнитное состояние, $Q U-$ квадрупольную фазу, $S S$ - сверхтвердую магнитную фазу, $I N$ - пространственно-неоднородное состояние. $D^{*}=2 J_{0}$ $-\tilde{J}_{0} / 2[33,34]$. Сплошные линии обозначают фазовые переходы первого рода, штриховая линия - фазовый переход второго рода.

мент отличен от нуля при $H \gtrsim H_{Q U}^{C}$, но все еще слишком мал из-за сильного влияния большой легкоплоскостной одноионной анизотропии. Это приводит к тому, что влияние магнитодипольного взаимодействия пренебрежимо мало вблизи линии устойчивости квадрупольной фазы. Поэтому аналитический анализ области устойчивости пространственно-неоднородной фазы вблизи $H_{Q U}^{C}$ невозможен. Этот анализ можно провести только численно, но это выходит за рамки настоящего аналитического исследования.

Используя полученные результаты, можно построить качественную фазовую диаграмму рассматриваемой системы (рис. 3). Пунктирная линия на рис. 3 обозначает фазовый переход второго рода из сверхтвердой магнитной фазы в ферромагнитную фазу, в то время как сплошные линии обозначают фазовые переходы первого рода. Необходимо напомнить, что мы рассматриваем случай большой легкоплоскостной одноионной анизотропии, энергия которой превосходит энергию обменного взаимодействия, что приводит к реализации квадрупольной фазы в слабом внешнем магнитном поле. Минимальное значение константы анизотропии, позволяющее реализоваться квадрупольной фазе, было найдено, например в $[33,34]: D^{*}=2 J_{0}-\tilde{J}_{0} / 2$. При меньших значениях константы одноионной анизотропии в системе может реализоваться антиферромагнитная или квадрупольно-антиферромагнитная фаза, но мы не рассматриваем этот случай.

\section{5. Заключение}

В работе исследована двумерная двухподрешеточная модель Изинга с ферромагнитным внутриподрешеточным и антиферромагнитным межподрешеточным обменными взаимодействиями и большой легкоплоскостной одноионной анизотропией. Показано, что в сильных магнитных полях подрешетки становятся эквивалентными, и в системе реализуется ферромагнитное состояние, характеризуемое векторным параметром порядка - намагниченностью. В случае слабого внешнего магнитного поля система переходит в квадрупольное состояние с нулевой намагниченностью, описываемое тензорным параметром порядка. В рассматриваемом случае учет магнитодипольного взаимодействия не влияет на динамику системы, проявляясь лишь в статической перенормировке энергетических уровней магнитного иона, а также в виде аддитивного слагаемого в линиях устойчивости однородных состояний (9) и (13). Однако эти аддитивные слагаемые могут быть довольно большими для некоторых редкоземельных сплавов [61,62].

Показано, что при произвольных соотношениях между зеемановской энергией и энергией анизотропии антиферромагнитное упорядочение между узлами различных подрешеток приводит к возникновению сверхтвердой магнитной фазы, т.е. углы ориентации магнитных моментов различных подрешеток различны и определяются системой уравнений (15). При этом магнитодипольное взаимодействие проявляется как в статической перенормировке этих углов, усиливая влияние легкоплоскостной одноионной анизотропии, так и приводит к реализации в системе пространственно-неоднородного состояния. Пространственно-неоднородное состояние может реализоваться лишь при выполнении условий (20), в противном случае система находится в монодоменном состоянии. Также найдены линия устойчивости (22) и период неоднородности вблизи линии фазового перехода из сверхтвердой фазы в пространственно-неоднородную фазу.

Необходимо отметить, что теоретические результаты, полученные в настоящей работе, качественно согласуются с недавними экспериментальными наблюдениями [4], в которых были обнаружены следующие фазы:

a) фазы с различными значениями проекций магнитного момента для различных подрешеток, показанные на рис. $2, b$ и 5 в [4]. Данным состояниям в нашем случае соответствует сверхтвердая магнитная фаза;

б) спиновое состояние с $\left|m_{z}\right\rangle=0$, показанное на рис. 2, $a$ и 5 в [4]; в нашем случае соответствующее квадрупольной фазе;

в) и, наконец, ферромагнитная и пространственнонеоднородная фазы, показанные на рис. 7 в [4].

Все фазовые состояния, перечисленные выше, наблюдались экспериментально в вырожденном спинорном бозе-газе ионов ${ }^{87} \mathrm{Rb}$. 


\section{Список литературы}

[1] E. Kim, M.H.W. Chan. Nature 427, 225 (2004).

[2] D.Y. Kim, M.H.W. Chan. Phys. Rev. Lett. 109, 155301 (2012).

[3] T. Matsuda, T. Tsuneto. Suppl. Prog. Theor. Phys. 46, 411 (1970).

[4] M. Vengalattore, J. Guzman, S.R. Leslie, F. Serwane, D.M. Stamper-Kurn. Phys. Rev. A 81, 053612 (2010).

[5] Y. Murakami, R. Oka, H. Aoki. Phys. Rev. B 88, 224404 (2013).

[6] J. Ye, Y. Chen. Nuclear Physics B 869 [FS], 242 (2013).

[7] D. Rossini, V. Giovannetti, R. Fazio. Phys. Rev. B 83, 140411(R) (2011).

[8] T. Giamarchi, C. Rüegg, O. Tchernyshyov. Nature Phys. 4, 198 (2008).

[9] T. Nikuni, M. Oshikawa, A. Oosawa, H. Tanaka. Phys. Rev. Lett. 84, 5868 (2000).

[10] G. Misguich, M. Oshikawa. J. Phys. Soc. Jpn. 73, 3429 (2004).

[11] Kwai-Kong Ng, T.K. Lee. Phys. Rev. Lett. 97, 127204 (2006).

[12] Y.A. Fridman, O.A. Kosmachev, P.N. Klevets. Eur. Phys. J. B 81, 185 (2011).

[13] P. Sengupta, C.D. Batista. Phys. Rev. Lett. 98, 227201 (2007).

[14] N. Laflorencie, F. Mila. Phys. Rev. Lett. 99, 027202 (2007).

[15] J.-D. Picon, A.F. Albuquerque, K.P. Schmidt, N. Laflorencie, M. Troyer, F. Mila. Phys. Rev. B 78, 184418 (2008).

[16] Pochung Chen, Chen-Yen Lai, Min-Fong Yang. Phys. Rev. B 81, 020409(R) (2010).

[17] A.F. Albuquerque, N. Laflorencie, J.-D. Picon, F. Mila. Phys. Rev. B 83, 174421 (2011)

[18] K.P. Schmidt, J. Dorier, A.M. Läuchli, F. Mila. Phys. Rev. Lett. 100, 090401 (2008).

[19] D. Yamamoto, I. Danshita. Phys. Rev. B 88, 014419 (2013).

[20] J.P. Renard, M. Verdaguer, L.P. Regnault, W.A.C. Erkelens, J. Rossat-Mignod, J. Ribas, W.G. Stirling, C. Vettier. J. Appl. Phys. 63, 3538 (1988).

[21] M. Orendáč, A. Orendáčová, J. Černák, A. Feher, P.J.C. Signore, M.W. Meisel, S. Merah, M. Verdaguer. Phys. Rev. B 52, 3435 (1995).

[22] Y. Tanaka, H. Tanaka, T. Ono, A. Oosawa, K. Morishita, K. Iio, T. Kato, H.A. Katori, M.I. Bartashevich, T. Goto. J. Phys. Soc. Jpn. 70, 3068 (2001).

[23] B. Dorner, D. Visser, U. Steigenberger, K. Kakurai, M. Steiner. Zeitschrift Phys. 72, 487 (1988).

[24] A. Harrison, D. Visser. J. Phys.: Condens. Matter 4, 6977 (1992).

[25] M. Steiner, K. Kakurai, W. Knop, B. Dorner, R. Pynn, U. Happek, P. Day, G. McLeen. Solid State Commun. 38, 1179 (1981).

[26] D. Ueltschi. Phys. Rev. E 91, 042132 (2015).

[27] D. Ueltschi. J. Mathematic. Phys. 54, 083301 (2013).

[28] L. Balents. Nature 464, 199 (2010).

[29] J. Romhányi, F. Pollmann, K. Penc. Phys. Rev. B 84, 184427 (2011).

[30] S. Miyahara, N. Furukawa. J. Phys. Soc. Jpn. 80073708 (2011).

[31] D. Peters, I.P. McCulloch, W. Selke. Phys. Rev. B 79, 132406 (2009).

[32] L. Seabra, N. Shannon. Phys. Rev. B 83, 134412 (2011).

[33] Ph.N. Klevets, O.A. Kosmachev, Yu.A. Fridman. JMMM 330, 91 (2013).

[34] Ph.N. Klevets, O.A. Kosmachev, Yu.A. Fridman. JMMM 348, 68 (2013).
[35] Б.А. Иванов. ФНТ 31, 841 (2005).

[36] Y. Eto, H. Saito, T. Hirano. Phys. Rev. Lett. 112, 185301 (2014).

[37] D. Jaksch, J.I. Cirac, P. Zoller, S.L. Rolston, R. Côté, M.D. Lukin. Phys. Rev. Lett. 85, 2208 (2000).

[38] D. DeMille. Phys. Rev. Lett. 88, 067901 (2002).

[39] A. Micheli, G.K. Brennen, P. Zoller. Nature Phys. 2, 341 (2006).

[40] R.P. Erickson, D.L. Mills. Phys. Rev. B 46, 861 (1992).

[41] Yu.A. Fridman, D.A. Matunin, Ph.N. Klevets, O.A. Kosmachev. JMMM 321, 3782 (2009).

[42] В.Г. Барьяхтар, Б.А. Иванов. ЖЭТФ 72, 1504 (1977).

[43] R. Antos, Y. Otani, J. Shibata. J. Phys. Soc. Jpn. 77, 031004 (2008).

[44] В.Г. Барьяхтар, Б.А. Иванов, М.В. Четкин. УФН 146, 417 (1985).

[45] Б.А. Иванов, Г.Г. Аванесян, А.В. Хвальковский, Н.Е. Кулагин, К.Э. Заспел, К.А. Звездин. Письма в ЖЭТФ 91, 190 (2010).

[46] B.A. Ivanov, A.K. Kolezhuk, G.M. Wysin. Phys. Rev. Lett. 76, 511 (1996)

[47] E.G. Galkina, A.Yu. Galkin, B.A. Ivanov, F. Nori. Phys. Rev. B 81, 184413 (2010).

[48] С.В. Малеев. ЖЭТФ 70, 2374 (1976).

[49] Yu.A. Fridman, D.V. Spirin. Phys. Status Solidi B 231, 165 (2002).

[50] Yu.A. Fridman, D.V. Spirin, C.N. Alexeyev, D.A. Matiunin. Eur. Phys. J. B 26, 185 (2002).

[51] Yu.A. Fridman, D.V. Spirin, Ph.N. Klevets. JMMM 253, 105 (2002).

[52] В.В. Вальков. ТМФ 76, 766 (1988).

[53] Р.О. Зайцев. ЖЭТФ 68, 207 (1975).

[54] Ю.Н. Мицай, Ю.А. Фридман. ТМФ 81, 1194 (1989).

[55] В.В. Вальков, Т.А. Валькова, С.Г. Овчинников. ЖЭТФ 88, 550 (1985)

[56] K.W.H. Stevens. Proc. Phys. Soc. A 65, 209 (1952).

[57] H.H. Chen, P.M. Levy. Phys. Rev. B 7, 4267 (1973).

[58] Yu.A. Fridman, O.A. Kosmachev, Ph.N. Klevets. JMMM 320, 435 (2008).

[59] В.В. Вальков, Т.А. Валькова. ЖЭТФ 99, 1881 (1991).

[60] C. Kittel. Quantum theory of solids. Wiley, N.Y. (1987). $523 \mathrm{p}$.

[61] Magnetic Properties of Rare Earth Metals / Ed. R.J. Elliott. Plenum Press, London (1972). 425 p.

62] P. Campbell. Permanent Magnet Materials and Their Application (Cambridge Studies in Magnetism). University Press, Cambridge (1996). 207 p. 